\title{
Intoxicações por plantas e micotoxinas associadas a plantas em bovinos no Rio Grande do Sul: 461 casos $^{1}$
}

\begin{abstract}
Daniel R. Rissi ${ }^{2}$, Raquel R. Rech ${ }^{2}$, Felipe Pierezan ${ }^{3}$, Adriane L. Gabriel ${ }^{4}$, Maria E. Trost ${ }^{4}$, Juliana S. Brum ${ }^{3}$, Glaucia D. Kommers ${ }^{5}$ e Claudio S.L. Barros ${ }^{5 *}$

ABSTRACT.- Rissi D.R., Rech R.R., Pierezan F., Gabriel A.L., Trost M.E., Brum J.S., Kommers G.D. \& Barros C.S.L. 2007. [Plant and plant-associated mycotoxins poisoning in cattle in Rio Grande do Sul, Brazil: 461 cases.] Intoxicações por plantas e micotoxinas associadas a plantas em bovinos no Rio Grande do Sul: 461 casos. Pesquisa Veterinária Brasileira 27(7):261-268. Departamento de Patologia, Universidade Federal de Santa Maria, 97105-900 Santa Maria, RS, Brazil. E-mail: claudioslbarros@uol.com.br

From 1990 to 2005, tissues from 2,912 cattle necropsies were examined at the Laboratory of Veterinary Pathology (LPV) of the Federal University of Santa Maria (UFSM), Brazil. These tissues came from necropsies performed by faculty members of the LPV or were mailedin samples from necropsy performed by veterinarian practitioners. In 461 (15.83\%) of these necropsies the cause of death was attributed to the ingestion of poisonous plants. In decreasing order of frequency poisoning by the following plants were registered: Senecio spp (56.14\%), Pteridium aquilinum (12.06\%), Ateleia glazioviana (10.31\%), Solanum fastigiatum (5.04\%), Baccharis coridifolia (3.29\%), Xanthium cavanillesii (3.07\%), Senna occidentalis (2.63\%), Ramaria flavo-brunnescens (2.41\%), Amaranthus spp (2.19\%), Vicia villosa (1.54\%), Ipomoea batatas, Prunus sellowii, cytrus pulp (0.44\% each), Cestrum parqui, Claviceps paspali, Claviceps purpurea, Brachiaria spp and Lantana sp (0.22\% each). In a given outbreak the number of affected cattle was substantially higher than the number of necropsies performed. The following aspects are discussed for each plant: geographical distribution; factors inducing ingestion; morbidity, mortality and lethality rates, clinical signs, necropsy findings, histopathology. For those plants in which information on the active principle and pathogenesis are available, these aspects are included in the discussion.
\end{abstract}

INDEX TERMS: Poisonous plants, epidemiology, cattle diseases.

RESUMO.- Foi realizado um levantamento nos arquivos do Laboratório de Patologia Veterinária (LPV) da Universidade Federal de Santa Maria (UFSM) e revisados os laudos de necropsias de bovinos realizadas entre 1990 e 2005 . Foram revisados 2.912 casos referentes a necropsias realizadas por membros do LPV ou a materiais de necropsias realizadas por

\footnotetext{
${ }^{1}$ Recebido em 22 de novembro de 2006.

Aceito para publicação em 20 de dezembro de 2006.

2 Programa de Pós-Graduação em Medicina Veterinária, área de concentração em Patologia Veterinária, Centro de Ciências Rurais, Universidade Federal de Santa Maria (UFSM), 97105-900, Santa Maria, Rio Grande do Sul.

${ }^{3}$ Bolsista de iniciação científica do CNPq, Departamento de Patologia, Laboratório de Patologia Veterinária (LPV), UFSM.

${ }^{4}$ Bolsista de aperfeiçoamento, LPV, UFSM.

5 Departamento de Patologia, UFSM. *Autor para correspondência: claudioslbarros@uol.com.br
}

veterinários de campo que enviaram amostras para avaliação histológica no LPV. Em 461 (15,83\%) das necropsias, a causa da morte foi atribuída à ingestão de plantas tóxicas. Em ordem decrescente de freqüência, intoxicações pelas seguintes plantas foram diagnosticadas: Senecio spp $(56,14 \%)$, Pteridium aquilinum (12,06\%), Ateleia glazioviana (10,31\%), Solanum fastigiatum (5,04\%), Baccharis coridifolia (3,29\%), Xanthium cavanillesii (3,07\%), Senna occidentalis $(2,63 \%)$, Ramaria flavobrunnescens (2,41\%), Amaranthus spp (2,19\%), Vicia villosa $(1,54 \%)$, Ipomoea batatas, Prunus sellowii e polpa cítrica $(0,44 \%$ cada), Cestrum parqui, Claviceps paspali, Claviceps purpurea, Brachiaria spp e Lantana sp (0.22\% cada). Em um determinado surto o número de bovinos afetados era substancialmente maior que o número de necropsias realizadas. São discutidos os aspectos relacionados à distribuição geográfica, fatores que induziram a ingestão, índices de morbidade, mortalidade e letalidade, sinais clínicos, achados de necropsia e histopatológicos para cada intoxicação. Quando conhecidos, foram incluídos na 
discussão aspectos relacionados ao princípio ativo e a patogenia da intoxicação.

TERMOS DE INDEXAÇÃO: Plantas tóxicas, epidemiologia, doenças de bovinos.

\section{INTRODUÇÃO}

Intoxicações por plantas são importante causa de morte de bovinos e de perdas diretas e indiretas no rebanho brasileiro. Casos desse tipo compõem aproximadamente $10 \%$ dos diagnósticos realizados em bovinos no Laboratório Regional de Diagnóstico (LRD) da Universidade Federal de Pelotas (UFPEL) e aproximadamente $14 \%$ dos diagnósticos realizados na Universidade Estadual de Santa Catarina (Riet-Correa \& Medeiros 2001). Apesar do extensivo estudo e da vasta literatura relacionada às plantas tóxicas do Brasil (Tokarnia et al. 1979, Riet-Correa et al. 1993, Riet-Correa et al. 1994, Tokarnia et al. 2000) há uma carência de informações em relação à frequiência das intoxicações por plantas nas várias regiões do país (Riet-Correa \& Medeiros 2001). Desse modo, julgamos importante a documentação dos casos de intoxicação por plantas em bovinos diagnosticados no LPV/ UFSM.

O objetivo deste trabalho é descrever os dados epidemiológicos relacionados à prevalência, incidência e distribuição geográfica dos casos de morte por intoxicações por plantas em bovinos no Rio Grande do Sul diagnosticados em 461 necropsias realizadas no LPV/UFSM, no período de 1990-2005. Adicionalmente serão abordados aspectos clinicopatológicos dos casos e sua importância como causa de morte de bovinos.

\section{MATERIAL E MÉTODOS}

Foram revisados todos os laudos de necropsias de bovinos realizadas pela equipe do LPV/UFSM ou por veterinários de campo que enviaram material para exame histopatológico no LPV durante 16 anos (19902005). Foram analisados todos os casos com diagnóstico de intoxicação por plantas e, desses protocolos, foram registradas as informações referentes à epidemiologia, aos sinais clínicos, achados de necropsia e histopatológicos. Para o registro foram considerados os diagnósticos morfológicos e os comentários feitos nos laudos de necropsia ou histopatológicos. Materiais de bovinos provenientes de outros estados e de experimentos não foram inclú́dos no estudo. Casos anteriores a 1990 também não foram incluídos devido à carência de informações necessárias para o desenvolvimento do trabalho.

\section{RESULTADOS}

Foram revisados 2.912 laudos de necropsia de bovinos nos arquivos do LPV/UFSM, que correspondem a material de necropsias realizadas pela equipe do LPV $(n=643)$ ou proveniente de necropsias realizadas por veterinários de campo que solicitaram exame histopatológico $(n=2.269)$. Desse total de casos de bovinos necropsiados, $461(15,83 \%)$ tinham diagnóstico de intoxicação por plantas. Esses bovinos eram provenientes de várias regiões do estado, mas houve maior prevalência de casos nas regiões Central, Noroeste, Missões e

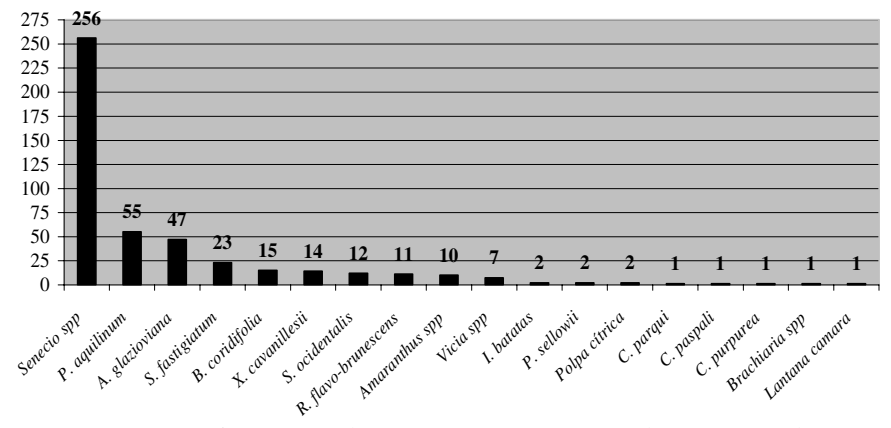

Fig.1. Intoxicações por plantas e micotoxinas diagnosticadas em bovinos no Laboratório de Patologia Veterinária, Universidade Federal de Santa Maria, Rio Grande do Sul, de 1990-2005.

Pampa do Rio Grande do Sul. Essa distribuição geográfica está relacionada à localização do LPV. Foram diagnosticados surtos ou casos isolados de intoxicação por 18 tipos de plantas ou micotoxicoses associadas a plantas. Os dados descritos para cada planta são pontuais, ou seja, foram coletados no momento da requisição dos exames, portanto podem não corresponder ao total de bovinos afetados em um determinado surto; o número de bovinos afetados foi, na maioria das vezes, substancialmente maior que o número de necropsias realizadas. $\mathrm{O}$ maior número de casos diagnosticados correspondeu à intoxicação por Senecio spp $(56,14 \%)$, seguido por Pteridium aquilinum (12,06\%), Ateleia glazioviana (10,31\%), Solanum fastigiatum $(5,04 \%)$ e Baccharis coridifolia $(3,29 \%)$. A relação completa dos diagnósticos de intoxicações por plantas e micotoxinas e suas frequiências estão na Figura 1. Dos 55 casos de intoxicação por $P$. aquilinum, 50 correspondiam à forma crônica. Desses, 46 eram caracterizados pelo desenvolvimento de carcinomas de células escamosas no trato digestivo e 4 por hematúria enzoótica; 5 casos correspondiam à forma aguda (doença hemorrágica). Nos 47 casos de intoxicação por A. glazioviana foram observados 44 casos de insuficiência cardíaca e desse total, 41 foram classificados como insuficiência cardíaca congestiva; os três casos restantes foram classificados como insuficiência cardíaca aguda. Em 10 desses laudos havia histórico de aborto concomitante ao quadro clínico cardíaco. A ocorrência de abortos como único achado foi descrita em duas ocasiões $(4,25 \%)$ e a forma neurológica da doença foi relatada em somente um caso $(2,12 \%)$. Os bovinos intoxicados por $A$. glazioviana eram proveniente das regiões Noroeste, Centro-Norte e Planalto Médio do Rio Grande do Sul. Os principais achados epidemiológicos, clínicos, de necropsia e histopatológicos registrados no laudos referentes ao período estudado estão nos Quadros 1 e 2 .

\section{DISCUSSÃO}

O diagnóstico de intoxicação por plantas nos casos revisados foi realizado com base na epidemiologia, nos sinais clínicos, nos achados de necropsia e histopatológicos, associados à presença da planta na pastagem ou ao histórico de ingestão da planta pelos bovinos afetados. A maioria desses dados pode ser obtida detalhadamente em trabalhos realizados pela equipe do LPV/UFSM (Barros et al. 1987a,b,c, 1990, 1992, 1999, 
Quadro 1. Dados epidemiológicos referentes aos casos de intoxicação por plantas e micotoxinas associadas a plantas em bovinos, diagnosticados no Laboratório de Patologia Veterinária, Universidade Federal de Santa Maria, no período de 1990-2005

\begin{tabular}{|c|c|c|c|c|c|c|c|}
\hline Plantas & $\begin{array}{c}\text { № de casos } \\
(\%)\end{array}$ & Idade & $\begin{array}{l}\text { Época } \\
\text { do ano }\end{array}$ & $\begin{array}{c}\text { Morbidade } \\
\text { (\%) }\end{array}$ & $\begin{array}{c}\text { Mortalidade } \\
(\%)\end{array}$ & $\begin{array}{c}\text { Letalidade } \\
(\%)\end{array}$ & $\begin{array}{l}\text { Evolução } \\
\text { clínica }\end{array}$ \\
\hline Senecio spp & $256(56,14)$ & a3 meses-12 anos & Ago-dez & $0,05-25$ & $0,05-25$ & 100 & 1 dia-2 anos \\
\hline Pteridium aquilinum & $\begin{array}{l}\mathrm{FC}^{\mathrm{b}}: 50(90,9) \\
\mathrm{FA}^{\mathrm{c}}: 5(9,09)\end{array}$ & $\begin{array}{l}\text { FC: } 3-24 \text { anos } \\
\text { FA: } 2 \text { anos }\end{array}$ & Todo o ano & $\begin{array}{c}\text { FC: } 5-9,6 \\
\text { FA: } 9,6\end{array}$ & $\begin{array}{c}\text { FC: } 1,8-9,6 \\
\text { FA: } 9,6\end{array}$ & $\begin{array}{c}\text { FC: } 50-100 \\
\text { FA: } 100\end{array}$ & $\begin{array}{c}\text { FC: } 1-18 \text { meses } \\
\text { FA: } 3 \text { dias }\end{array}$ \\
\hline Ateleia glazioviana & $\begin{array}{c}\text { IC }^{\mathrm{d}}: 44(93,61) \\
\text { Abortos: } 2(4,25) \\
\text { FN }^{\mathrm{e}}: 1(2,12)\end{array}$ & 1-12 anos & Abr-ago & $5-46,1$ & $4-33,3$ & $19-100$ & - \\
\hline Solanum fastigiatum & $23(5,04)$ & $1-8$ anos & Fev-dez & $6,6-9,6$ & $6,6-14,2$ & $25-100$ & 1-18 meses \\
\hline Baccharis coridifolia & $15(3,29)$ & 1-4 anos & Ago-dez & $2,5-71,4$ & $6,4-71,4$ & 100 & 1-3 dias \\
\hline Xanthium cavanillesii & $14(3,07)$ & 6 meses- 2 anos & Ago-out & $0,6-18$ & $0,6-13,2$ & $66-100$ & 24 horas \\
\hline Senna occidentalis & $12(2,63)$ & 8 meses-5 anos & Abr-set & $4,2-66$ & $2,8-66$ & $50-100$ & $\begin{array}{c}12 \text { horas-2 } \\
\text { semanas }\end{array}$ \\
\hline Ramaria flavo-brunnescens & $11(2,41)$ & $1-1,5$ anos & Abr & $4,4-50$ & $2,4-25$ & $50-67$ & $8-15$ dias \\
\hline Amaranthus spp & $10(2,19)$ & 2-6 anos & Fev-abr & $5,3-32$ & 5,3-33 & $90-100$ & 3-7 dias \\
\hline Vicia spp & $7(1,54)$ & 5-12 anos & Ago-dez & $3,33-16,6$ & $3,84-16,6$ & $50-100$ & $10-30$ dias \\
\hline Ipomoea batatas & $2(0,44)$ & 1-7 anos & Set & 21,7 & 17,3 & 80 & 1-4 dias \\
\hline Prunus sellowii & $2(0,44)$ & 1-10 anos & Mai-jul & - & - & 100 & súbita \\
\hline Polpa cítrica & $2(0,44)$ & 1 ano & Dez & - & - & - & $2-3$ semanas \\
\hline Cestrum parqui & $1(0,22)$ & 6 meses & Set & - & - & 100 & ２4 horas \\
\hline Claviceps paspali & $1(0,22)$ & 10 meses- 2 anos & Abr & 8 & - & - & 2 dias \\
\hline Claviceps purpurea & $1(0,22)$ & adultos & Dez-fev & 56 & 0 & 0 & 2-3 meses \\
\hline Brachiaria sp & $1(0,22)$ & $1-2$ anos & Mai-jun & - & - & - & - \\
\hline Lantana $\mathrm{sp}$ & $1(0,22)$ & 1,5 ano & $\mathrm{Abr}$ & 10 & 2 & 20 & não informado \\
\hline
\end{tabular}

a Intoxicação por Senecio spp ocorre em bovinos adultos em pastoreio, porém em um surto foram afetados bezerros que ingeriram feno contaminado pela planta (Barros et al. 2007); ${ }^{b}$ forma crônica (Carcinoma de células escamosas do trato alimentar e hematúria enzoótica); ${ }^{\mathrm{c}}$ forma aguda; ${ }^{\mathrm{d}}$ insuficiência cardíaca; eforma neurológica.

2001, 2006, 2007, Barros 1993, 1998, Barth et al. 1994, Driemeier \& Barros 1991, Driemeier et al. 1999, Fighera \& Barros 2004, Fighera et al. 2003, 2005, 2006, Garmatz et al. 2001, Gava \& Barros 2001, Ilha et al. 2001, 2003, Langohr et al. 2005, Lemos et al. 1993, Masuda et al. 2005, Peixoto et al. 2003, Rech et al. 2004, 2006, 2007, Riet-Correa et al. 2004, Rissi et al. 2005, 2007, Souto et al. 2006a,b, Souza \& Graça 1993, Torres et al. 1997).

Foi verificado que no período estudado aproximadamente $16 \%$ dos casos de morte de bovinos diagnosticados no LPV/ UFSM foram atribuídos à ingestão de plantas tóxicas. Esse percentual é semelhante ao observado por outros laboratórios de diagnóstico do Rio Grande do Sul e de outros estados. Intoxicações por plantas em bovinos compõem aproximadamente $10 \%$ dos casos diagnosticados no LRD/UFPEL e aproximadamente $14 \%$ dos casos na Universidade Estadual de Santa Catarina (Riet-Correa \& Medeiros 2001). Dados do Setor de Patologia Veterinária (SPV) da Universidade Federal do Rio Grande do Sul (UFRGS) indicam que aproximadamente 7\% das mortes de bovinos são causadas pela ingestão de plantas tóxicas na área de influência do SPV (Pedroso et al. 2005). A mortalidade anual estimada de bovinos no estado é de $5 \%$ (Riet-Correa \& Medeiros 2001). A partir desses dados pode-se concluir que a intoxicação por plantas é uma das principais causas de morte de bovinos no Rio Grande do Sul e um dos principais fatores de perdas econômicas no estado. Considerando-se uma população total de bovinos de aproximadamente 14,5 milhões de cabeças (MAPA 2006) e um índice médio de mortalidade por plantas tóxicas de $14,5 \%$, conclui-se que aproximadamente 105 mil bovinos morrem anualmente em decorrência de intoxicação por plantas no Rio Grande do Sul, o que resulta num prejuízo aproximado de US\$ 21 milhões por ano (considerado um valor médio de US\$ 200 por animal).

Dentre os casos de intoxicação por plantas diagnosticados no LPV/UFSM, 56,14\% foram atribuídos à intoxicação por espécies do gênero Senecio. Esse índice demonstra que a intoxicação pelos alcalóides pirrolizidínicos (AP) presentes nessas plantas foi a principal causa de morte em bovinos nesse período. Dados dos nossos arquivos nesses 16 anos indicam que nenhuma outra doença de bovinos, independente da causa, apresentou prevalência igual ou superior à intoxicação por Senecio spp e é provável que o número de casos dessa intoxicação seja ainda maior, já que vários surtos são comunicados pelos produtores ou veterinários sem o envio de material para exame, semelhantemente ao que ocorre em outras regiões do Estado (Barros et al. 1992, Schild 2006). Intoxicação por Senecio spp foi responsável por 64,86\% dos diagnósticos de intoxicação por plantas em bovinos no SPV/ UFRGS (Pedroso et al. 2005). Surtos de intoxicação por Senecio spp ocorridos no sul do estado foram avaliados em um levantamento feito no LRD/UFPEL (Karam et al. 2004) e esses autores informam um aumento no número de casos de intoxicação por Senecio spp nos últimos anos, situação semelhante à que foi observada na área de influência do nosso laboratório. Alguns fatores que podem ter contribuído para esse crescimento foram a diminuição do rebanho de ovinos e certas alterações climáticas, como longos períodos de seca, situações observadas no estado durante esse período (Karam et al. 2004). Os ovinos são mais resistentes à intoxicação por 
Quadro 2. Principais achados clínicos, de necropsia e histopatológicos dos casos de intoxicação por plantas e micotoxinas associados a plantas em bovinos, diagnosticados no Laboratório de Patologia Veterinária, Universidade Federal de Santa Maria, de 1990-2005

\begin{tabular}{|c|c|}
\hline Plantas & Sinais clínicos \\
\hline Senecio spp & $\begin{array}{l}\text { Apatia, salivação, emagrecimento progressivo, tenesmo, } \\
\text { prolapso retal, diarréia escura, tremores, incoordenação, } \\
\text { agressividade, cegueira, febre; raramente icterícia e } \\
\text { fotodermatite. }\end{array}$ \\
\hline Pteridium aquilinum & $\begin{array}{l}\mathrm{FC}^{\mathrm{a}} \text { : }\left(\mathrm{TTD}^{\mathrm{b}}\right) \text { emagrecimento progressivo, tosse, timpanismo, } \\
\text { regurgitação; (HE) })^{\mathrm{c}} \text { emagrecimento e urina escura. } \mathrm{FA}^{\mathrm{C}} \text { : } \\
\text { emagrecimento, diarréia com san-gue, febre, palidez e } \\
\text { presença de petéquias em mucosas, corrimento nasal e } \\
\text { vaginal sanguinolento, hemorragias em locais de punção } \\
\text { por agullha. }\end{array}$ \\
\hline Ateleia glazioviana & $\begin{array}{l}\text { ICC }{ }^{\mathrm{d}} \text { : edema subcutâneo ventral, emagrecimento } \\
\text { progressivo, ingurgitamento da jugular, diarréia, decúbito } \\
\text { e cansaço após movimentação. ICA }{ }^{\mathrm{e}} \text { : morte súbita, } \\
\text { principalmente após movimen-tação. FN } \text { f }^{\mathrm{f}} \text { depressão, } \\
\text { cegueira, andar cambaleante. }\end{array}$ \\
\hline
\end{tabular}

Solanum fastigiatum Hipermetria, tremores musculares, perda de equilíbrio, crises convulsivas; essas crises ocorrem principalmente quando os bovinos são movimentados.

Baccharis coridifolia Timpanismo, tremores, instabilidade dos membros pélvicos, fezes secas, polidipsia e inquietação.

Xanthium cavanillesii Anorexia, desidratação, tenesmo, incoordenação, agressividade, decúbito e cegueira.

Senna ocidentalis

Fraqueza muscular, tremores, incoor-denação dos membros pélvicos, diarréia, decúbito; os bovinos se mantêm alerta e com apetite normal.

Ramaria flavo- $\quad$ Emagrecimento, febre, palidez de mucosas, queda dos pêlos brunnescens vassoura da cauda, do estojo corneo dos chifres e cascos, sialorréia e alisamento da superficie da língua, com ulceração do epitélio.

Amaranthus spp Depressão, edema subcutâneo, descarga nasal serosa, emagrecimento progressivo, incoordenação e fezes com estrias de sangue.

Vicia spp

Febre, emagrecimento progressivo, prurido intenso, espessamento, enrrugamento e áreas alopécicas coalescentes na pele, descarga nasal serosa, queda na produção de leite e diarréia. Sinais semellhantes aos da intoxicação por polpa cítrica.

Ipomoea batatas Dispnéia, dilatação das narinas e exten-são do pescoço para frente.

Prunus sellowi

Desidratação, diarréia, andar cambaleante, palidez de mucosas.

Polpa cítrica

Áreas alopécicas e crostosas na pele do pescoço e acima do úbere, às vezes com exsudato sero-sanguinolento; palidez de mucosas, corrimento ocular seroso e fezes com estrias de sangue. Sinais semelhantes aos da intoxicação por Vicia spp.

Cestrum parqui Diarréia, desnutrição e decúbito.

Claviceps paspali

Tremores e quedas.

Claviceps purpure

elevada, anorexia, salivação, respiração ofegante e protrusão da língua; sinais clínicos intensificados durante o dia e os bovinos procuravam locais com sombra.

Brachiaria sp Anorexia, emagrecimento progressivo, desidratação, timpanismo, icterícia, diarréia e febre.

Lantana $\mathrm{sp}$

Achados de necropsia

Emaciação, edema subcutâneo ventral, ascite, edema de mesentério e das pregas do abomaso, distensão e edema da vesícula biliar, com pólipos na mucosa, figado firme, aumentado ou diminuído de tamanho, e com a cápsula acinzentada.

FC:(TTD)massastumoraisnotratodigestivo(basedalíngua, palato mole, faringe, esôfago, entrada do rúmen), freqüientemente associadas a papilomas; metástases no pulmão, linfonodos cervicais, retrofaríngeos, abdominais e figado; (HE): urina vermellha e tumores na mucosa da bexiga, podendo haver ulceração e acúmulo de fibrina na mucosa vesical. FA: hemorragias nas serosas de vários órgãos, intestinorepletodesangue,áreas de infartonosrinsefigado. ICC: edema subcutâneo ventral, hidroperitôneo, edema de mesentério e das pregas do abomaso; aumento de volume cardíaco e dilatação ventricular, com áreas brancas coalescentes no miocárdio.Aumento devolume e coloração escura do figado, com aspecto de nozmoscada. ICA: aumento de volume cardíaco, espessamento e áreas brancas e firmes no miocárdio. FN: ocasionalmente pequenas áreas brancas no miocárdio.

Maioria dos casos sem alterações; ocasional-mente pode haver atrofia cerebelar e lesões secundárias a traumatismos.

Desidratação, conteúdo líquido abundante no rúmen; avermelhamento e lesões erosivas na mucosa dos pré-estômagos.

Hemorragias na serosa de vários órgãos, edema das pregas do abomaso e da parede da vesícula biliar, fezes ressequidas; figado com acentuação do padrão lobular (áreas vermelhas deprimidas circundadas por halos claros) e hidropericárdio.

Áreas pálidas nos grandes grupos musculares do dorso e membros pélvicos, diafragma; alguns bovinos apresentam urina escura (mioglobinúria). Alisamento, com perda das papilas linguais e lesões erosivas e ulcerativas na superficie da língua e do esôfago.

Edema subcutâneo, hidroperitôneo; edema perirrenal e hematomas subcapsulares renais; tumefação e palidez dos rins; lesões ulcerativas no trato digestivo (uremia); hidropericárdio.

Múltiplos nódulos branco-acinzentados e firmes nas superfícies natural e de corte de vários órgãos. Lesões semelhantes às da intoxicação por polpa cítrica.

Pulmões armados e de consistência borrachenta.

Sem alterações.

Áreas puntiformes brancas nas superficies capsular e de corte de vários órgãos, com ou sem hemorragia. Lesões semelhantes às da intoxicação por polpa cítrica.

Hidroperitôneo, edema das pregas do abomaso; aumento de volume hepático e acentuação do padrão lobular (áreas vermelhas deprimidas circundadas por halos claros)

Sem alterações.

Enfisema pulmonar.

Icterícia e figado aumentado de volume.

Superfícies capsular e de corte hepáticas com áreas escuras intercaladas com áreas claras.

\author{
Achados histopatológicos
}

Perda da arquitetura hepática, com fibrose difusa proliferação de ductos biliares, megalocitose e nódulos de regeneração; proliferação adenomatosa da mucosa da vesícula biliar; encefalopatia hepática (degeneração esponjosa da substância branca). FC: (TTD) carcinomas de células escamosas e papilomas no trato digestivo; (HE): focos de hematopoese extramedular; cistite, hemangiomas e outros tumores na bexiga. FA: aplasia de medula, hemorragias intramurais e nas serosas de vários órgãos, áreas focais de necrose invadidas por bactérias, principalmente no figado e rim, trombos e áreas de necrose nos pulmões.

ICC: tumefação e necrose de fibras cardíacas, com deposição de tecido conjuntivo fibroso e infiltrado histiocitário. ICA: extensas áreas de tumefação e necrose de fibras cardíacas, com infiltrado mononuclear e de tecido conjuntivo multifocal. FN: fibrose cardíaca difusa associada a necrose de fibras; degeneração esponjosa na substância branca do encéfalo, principalmente na FN.

Degeneração microvacuolar citoplasmática e perda de células de Purkinje, com proliferação da glia de Bergmann; esferóides axonais na camada de células granulares e substância branca cerebelar; dimuição da camada molecular:

Degeneração e necrose do revestimento epitelial dos pré-estômagos e necrose do tecido linfóide.

Necrose hepatocelular centrolobular ou massiva, com hemorragia e degeneração gordurosa de hepatócitos remanescentes perilesionais.

Tumefação, degeneração segmentar, necrose flocular e regeneração de fibras musculares.

Atrofia das papilas linguais, desprendimento e necrose do epitélio da língua e do esôfago, com ulceração, infiltrado inflamatório neutrofilico e de fibrina e miríades de bactérias intralesionais. Degeneração, necrose e regeneração tubular renal, com proteinose tubular e fibrose intersticial.

Nódulos de macrófagos epitelióides e células gigantes multinucleadas, linfócitos, plasmócitos e eosinófilos (granulomas).

Pneumonia intersticial, com infiltrado de fibroblastos e células inflamatórias, hiperplasia e hipertrofia de pneumócitos tipo-II, edema e enfisema. Sem alterações.

Infiltrado inflamatório granulomatoso multifocal e coalescente em vários órgãos; vacuolização das células epiteliais, hiperqueratose paraqueratótica, focos de necrose na junção derme/epiderme e infiltrado inflamatório perifolicular na derme superficial.

Necrose hepatocelular centrolobular ou massiva difusa, hemorragia e degeneração gordurosa de hepatócitos remanescentes.

Sem alterações.

Hipertrofia da musculatura lisa dos bronquíolos e ruptura de septos alveolares.

Bilestase e pericolangite histiocitária difusa, com macrófagos espumosos.

Retenção biliar hepática e nefrose tubular. 
AP e podem ser um bom meio de controle da planta, pois a removem do campo e não desenvolvem a doença (Tokarnia et al. 2000). Outro aspecto clínico dessa intoxicação é a ocorrência de distúrbios neurológicos decorrentes à insuficiência hepática, e que podem ser confundidos com os de outras doenças do sistema nervoso central (SNC) de bovinos, inclusive a raiva (Barros et al. 1992). Em um estudo retrospectivo de doenças do SNC de bovinos no Rio Grande do Sul, aproximadamente $43 \%$ dos casos de lesões degenerativas (status spongiosus) no encéfalo foram atribuídos à intoxicação por Senecio spp (Sanches et al. 2000). Em outro estudo, essas lesões foram observadas em aproximadamente $87 \%$ dos bovinos intoxicados por Senecio spp no Rio Grande do Sul (Masuda et al. 2005). Esses dados indicam que essa intoxicação também deve ser considerada no diagnóstico diferencial de doenças do SNC de bovinos.

Intoxicação por Pteridium aquilinum, Ateleia glazioviana, Solanum fastigiatum e Bacharis coridifolia foram descritas em um número significativo de casos durante o período avaliado. Intoxicação por $P$. aquilinum foi diagnosticada em 55 casos $(12,06 \%)$, dos quais 50 eram da forma crônica - 46 casos de carcinomas de células escamosas no trato digestivo e quatro casos de hematúria enzoótica - (Souza \& Graça 1993, Souto et al. 2006a,b) e 5 da forma aguda (Fighera et al. 2006). Esse diagnóstico teve um aumento substancial nos últimos três anos, devido à vigilância ativa do LPV para o estudo dos neoplasmas do trato digestivo superior e da bexiga em bovinos induzidos pela ingestão de P. aquilinum (Souto et al. 2006a,b). Isso indica que essa intoxicação pode ter sido subdiagnosticada ao longo dos anos, pois os bovinos afetados geralmente são provenientes de pequenas propriedades, onde os produtores são desprovidos dos meios necessários para o conhecimento da condição e apoio técnico para diagnóstico. Os tumores do trato digestivo foram descritos como a forma mais comum na intoxicação por $P$. aquilinum em Santa Catarina, onde é considerada a principal causa de morte relacionada ao consumo da planta (Gava 1993). A baixa incidência da forma aguda pode ser explicada pelo fato de que os bovinos precisam ingerir maior quantidade da planta em menor período de tempo para o desenvolvimento da doença, principalmente depois de roçadas, quando a planta está em fase de brotação (Barros et al. 1987a).

Intoxicação por $A$. glazioviana foi descrita principalmente em bovinos provenientes da região Noroeste, Centro-norte e Planalto Médio do Rio Grande do Sul e coincidem com a distribuição geográfica da planta (Tokarnia et al. 2000, Gava et al. 2001). Houve maior prevalência de casos da forma cardíaca $(93,61 \%)$, que é o quadro clínico mais freqüientemente desenvolvido pelos bovinos intoxicados (Gava et al. 2001). Os bovinos que desenvolvem insuficiência cardíaca podem desenvolver inicialmente sinais clínicos nervosos (Gava et al. 2001). Desse modo, os diagnósticos de intoxicação por $A$. glazioviana tendem a ser realizados na fase crônica da doença, tornando artificialmente baixo o número de casos de doença neurológica. Abortos foram relatados em 10 propriedades onde ocorreram os casos de insuficiência cardíaca associada ao consumo de $A$. glazioviana. Isso é comum na intoxicação por essa planta, onde as manifestações clínicas são variadas e podem ocorrer isoladas ou em conjunto durante um surto (Gava et al. 2001).

Apesar de descrita como uma planta de baixo interesse pecuário (Riet-Correa \& Méndez 1993) foram observados 23 casos $(5,04 \%)$ de intoxicação por $S$. fastigiatum e alguns desses surtos foram recentemente descritos (Rech et al. 2006). Os bovinos afetados estavam em campos altamente infestados pela planta. De modo semelhante ao que ocorre com a intoxicação por P. aquilinum, os bovinos intoxicados por $S$. fastigiatum também são provenientes de pequenas propriedades, o que, por razões econômicas, dificulta a chegada desses animais aos laboratórios de diagnóstico.

B. coridifolia é considerada a planta mais tóxica no Sul do Brasil e no Uruguai e uma única ingestão é capaz de matar um bovino em poucas horas (Riet-Correa \& Méndez 1993). Apesar dessa característica, a intoxicação por essa planta foi incriminada em somente 15 casos $(3,29 \%)$ no período estudado. Provavelmente isso seja devido ao fato de que a maioria dos casos não é relatada aos laboratórios de diagnóstico veterinário, pois os produtores e veterinários reconhecem a toxicidade dessa planta e prontamente programam medidas de controle (Rissi et al. 2005). Outra explicação é que bovinos nativos de locais onde a planta ocorre não a ingerem (Barros 1998), a não ser que sejam transferidos a áreas infestadas; nesse caso o risco de intoxicação aumenta consideravelmente. Apesar de a ocorrência da planta ser descrita na fronteira oeste do Rio Grande do Sul e no Uruguai (Tokarnia et al. 2000), descrições de surtos de intoxicação por $B$. coridifolia em bovinos em Vacaria (Rissi et al. 2005) e em ovinos em Caxias do Sul (Rozza et al. 2006) demonstram que a planta tem distribuição mais ampla do que a geralmente descrita.

Foram diagnosticados 14 casos $(3,07 \%)$ de intoxicação por Xanthium cavanillesii. Os achados clínicos, de necropsia e histopatológicos nesses casos são idênticos aos descritos no caso de intoxicação por Cestrum spp e essa é uma característica comum às plantas que causam doença hepática aguda em bovinos (Tokarnia et al. 2000, Rissi et al. 2007). Assim, como os sinais clínicos e a patologia das intoxicações por plantas hepatotóxicas de ação aguda são semelhantes, é importante que se faça o diferencial pelo achado da planta envolvida no pasto onde estão os animais (Rissi et al. 2007).

Os principais problemas relacionados à invasão de pastagens por plantas tóxicas incluem morte de bovinos, abortos, defeitos congênitos, baixo desempenho produtivo por doença crônica, subutilização de pastagens e altos custos com implantação de medidas de controle (James 1978, Culvenor 1984). Dentre esses prejuízos a morte de bovinos é um índice facilmente avaliado, enquanto que as outras perdas são de difícil mensuração (Dwyer 1978, Schuster 1978) e se tornam um problema constante nas propriedades. A experiência de outros países tem demonstrado que muitas dessas perdas podem ser evitadas ou minimizadas e que apenas ocasionalmente ocorrem problemas em locais onde práticas de manejo adequadas foram implantadas (Dwyer 1978, Schuster 1978, Riet-Correa \& Medeiros 2001). Boa quantidade de forragem e suplementação mineral são algumas das principais 
medidas a serem adotadas, já que a maioria das plantas tóxicas não é palatável e os bovinos raramente as consomem, exceto quando estão famintos ou apresentem carências nutricionais. Desse modo, deve-se evitar que bovinos sejam introduzidos em áreas desconhecidas sem antes fornecer alimento e água; animais famintos, principalmente após longas viagens e estresse são menos seletivos e mais propensos a ingerir plantas tóxicas. No Rio Grande do Sul isso é freqüientemente observado em casos de intoxicação por $B$. coridifolia (Rissi et al. 2005). Em relação à pastagem, o rebanho deve ser ajustado de modo que não haja superlotação na área. Sistemas de pastejo rotativo podem ser úteis para evitar o consumo de determinada planta em sua fase mais tóxica (Dwyer 1978, Schuster 1978, Riet-Correa \& Medeiros 2001). A eliminação dos espécimes tóxicos, mecanicamente ou quimicamente (Schuster 1978) e o pastoreio de espécies animais resistentes à intoxicação por determinada planta em sua fase mais tóxica (Riet-Correa \& Medeiros 2001) também podem ser empregados para minimizar a infestação por plantas tóxicas na pastagem. Outras formas de controle das intoxicações por plantas incluem a vacinação dos animais, controle biológico por inimigos naturais (principalmente insetos ou fungos), detoxificação microbiana do rúmen (isto é, adaptação gradativa da microflora ruminal aos princípios tóxicos presentes em determinada planta), aplicação de substâncias que neutralizam os princípios tóxicos (por ex., o carvão ativado), aversão alimentar condicionada e seleção de forrageiras ou grãos não-tóxicos ou menos tóxicos (Riet-Correa \& Medeiros 2001).

Medidas de controle não têm apresentado resultados satisfatórios no país e assim as intoxicações por plantas continuam causando perdas econômicas significativas. $\mathrm{O}$ estudo das plantas tóxicas normalmente segue a tecnificação dos sistemas de produção de pastagens, como ocorreu nos EUA e na Austrália (Whittem 1978) e o manejo das pastagens é citado como o meio mais econômico de prevenir as perdas ocasionadas pelas intoxicações por plantas (Schuster 1978). A alta pressão de pastejo em áreas de campo nativo pode levar a mudanças na composição botânica e isso pode aumentar o risco de intoxicação por plantas, pois há tendência de que as espécies mais palatáveis sejam reduzidas pelo consumo. As espécies menos palatáveis podem ser potencialmente tóxicas, mas não causarão problemas se não houver quantidade suficiente ou se os bovinos tiverem acesso a outro tipo de alimentação (Everist 1978). Por outro lado, a implantação de pastagens cultivadas pode reduzir o número de casos de intoxicações pela eliminação de espécies tóxicas nativas ou aumentar o risco de intoxicações caso o cultivar seja potencialmente tóxico (Everist 1978), como pôde ser constatado nos casos de intoxicação por Vicia spp.

A mortalidade de bovinos em áreas remotas de uma região impede ou dificulta a realização de necropsias, a não ser que haja um contato freqüente de veterinários no local, que podem fazer o diagnóstico ou contatar laboratórios interessados em documentar os casos (Whittem 1978). Essa situação é semelhante ao que ocorreu nos casos de intoxicação por P. aquilinum e $S$. fastigiatum neste estudo, onde o LPV/UFSM, pela constante comunicação com veterinários locais, realiza uma vigilância ativa em relação a casos suspeitos de intoxicação por plantas.

Apesar de algumas plantas causarem um quadro clinicopatológico típico, outras não apresentam achados específicos ou não produzem nenhuma alteração (Tokarnia et al. 2000); nesses casos é importante que seja realizada uma busca minuciosa de evidências que comprovem o consumo da planta no campo ou mesmo procurar pela planta no trato digestivo do animal (Whitem 1978). Isso ocorre em casos de intoxicação por Claviceps paspali e Prunus sellowii, onde o quadro clínico, a presença e o consumo da planta fornecem evidências para o diagnóstico, porém os bovinos intoxicados não desenvolvem alterações de necropsia ou histopatológicas relacionadas à doença. Nesses casos a busca de informações epidemiológicas é essencial para o diagnóstico (Riet-Correa et al. 1983, Tokarnia et al. 2000).

Apesar da vasta informação e trabalho realizado sobre intoxicações por plantas em bovinos no Brasil (Tokarnia et al. 1979, Riet-Correa et al. 1993, Tokarnia et al. 2000), não existe nenhum programa oficial com objetivo de controlar e minimizar as perdas ocasionadas por esse problema no rebanho bovino brasileiro. Além disso, nota-se uma carência de informações referente à incidência de intoxicações por plantas no país (Riet-Correa \& Medeiros 2001). A falta de informação de veterinários e produtores foi apontada como um dos principais fatores que contribuem para o crescimento do número de casos de intoxicação por plantas numa determinada região (James 1978). Na rotina do LPV/UFSM nota-se que essa falta de informação conduz a dúvidas e, muitas vezes, ao ceticismo de alguns produtores frente a um diagnóstico de intoxicação por plantas em um rebanho. A intoxicação por plantas constitui um dos principais problemas para o rebanho bovino do Rio Grande do Sul e provavelmente esse problema se estenda para a maioria das regiões do Brasil. Ao exemplo do que ocorre com outros programas oficiais do Ministério da Agricultura Pecuária e Abastecimento, é neces-sário um sistema de controle e de geração de informações referentes às espécies de plantas tóxicas em cada região do país e cujo foco fossem os veterinários de campo e produtores, tentando, dessa forma, minimizar os prejuízos agropecuá-rios que há muito tempo se refletem na rotina de vários laboratórios de diagnóstico veterinário.

\section{REFERÊNCIAS}

Barros C.S.L. 1993. Intoxicação por Senna ocidentalis, p.201-213, In: Riet-Correa F., Méndez M.C. \& Schild A.L. (ed.), Intoxicações por Plantas e Micotoxicoses em Animais Domésticos. Editorial Agropecuária Hemisfério Sur, Montgevideo. 340p.

Barros C.S.L. 1998. Livestock poisoning by Baccharis coridifolia, p.569-572. In: Garland T. \& Barr A.C. (ed.), Toxic Plants and Other Natural Toxicants. $\mathrm{CAB}$ International, Wallingford. 576p.

Barros C.S.L., Graça D.L., Santos M.N. \& Barros S.S. 1987a. Intoxicação aguda por samambaia (Pteridium aquilinum) em bovinos no Rio Grande do Sul. Hora Vet., Porto Alegre, 37:33-39.

Barros C.S.L., Metzdorf L.L. \& Peixoto P.V. 1987b. Ocorrência de surtos da intoxicação por Senecio brasiliensis (Compositae) em bovinos no Rio Grande do Sul. Pesq. Vet. Bras. 7:101-107.

Barros S.S., Riet-Correa F., Andujar M.B., Barros C.S.L., Méndez M.C. \& Schild 
A.L. 1987c. Solanum fastigiatum var, fastigiatum and Solanum sp. poisoning in cattle: ultrastructural changes in the cerebellum. Pesq. Vet. Bras. 7:1-5.

Barros C.S.L., Pilati C., Andujar M.B., Graça D.L., Irigoyen L.F., Lopes S.T. \& Santos C.F. 1990. Intoxicação por Cassia occidentalis (Leg. Caes.) em bovinos. Pesq. Vet. Bras. 10:47-58.

Barros C.S.L., Driemeier D., Pilati C., Barros S.S. \& Castilhos L.M. 1992. Senecio spp. poisoning in cattle in southern Brazil. Vet. Human Toxicol. 34:241246.

Barros C.S.L., Ilha M.R.S., Bezerra P.S., Langohr I.M. \& Kommers G.D. 1999. Intoxicação por Senna occidentalis (Leg. Caesalpinoideae) em bovinos em pastoreio. Pesq. Vet. Bras. 19:68-70.

Barros C.S.L., Fighera R.A., Rozza D.B., Rech R.R., Sallis S.V. \& Langohr I.M. 2001. Doença granulomatosa sistêmica em bovinos no Rio Grande do Sul associada ao pastoreio de ervilhaca (Vicia spp). Pesq. Vet. Bras. 21:162171.

Barros R.R., Irigoyen L.F., Kommers G.D., Rech R.R., Fighera R.A. \& Barros C.S.L. 2006. Intoxicação por Ramaria flavo-brunnescens (Clavariaceae) em bovinos. Pesq. Vet. Bras. 26:87-96.

Barros C.S.L., Castilhos L.M.L., Rissi D.R., Kommers G.D. \& Rech R.R. 2007. Biópsia hepática no diagnóstico da intoxicação por Senecio brasiliensis em bovinos. Pesq. Vet. Bras. 27(1):53-60.

Barth A.T., Kommers G.D., Salles M.S., Wouters F. \& Barros C.S.L. 1994. Coffee senna (Senna occidentalis) poisoning in cattle in Brazil. Vet. Human Toxicol. 36:541-545.

Culvenor C.C.J. 1984. Economic loss due to poisonous plants in Australia, p.3-13. In: Seawright A.A., Hegarty M.P., James L.F. \& Keeler R.F. (ed.), Plant Toxicology. Dominion, Melbourne. 624p.

Driemeier D. \& Barros C.S.L. 1992. Intoxicação experimental por Senecio oxyphyllus (Compositae) em bovinos. Pesq. Vet. Bras. 12:33-42.

Driemeier D., Barros C.S.L. \& Pilati C. 1991. Seneciose em bovinos. Hora Vet., Porto Alegre, 10:23-30.

Driemeier D., Irigoyen L.F., Loretti A.P., Colodel E.M. \& Barros C.S.L. 1999. Intoxicação espontânea pelos frutos de Xanthium cavanillesii (Asteraceae) em bovinos. Pesq. Vet. Bras. 19:12-18.

Dwyer D.D. 1978. Impact of poisonous plants on western U.S. grazing systems and livestock operations, p.13-21. In: Keeler R., Van Kampen K.R. \& James L.F. (ed.), Effetcs of Poisonous Plants on Livestock. Academic Press, New York. 600p.

Everist S.L. 1978. Effect of land use on plant poisoning of livestock in Australia, p.47-56. In: Keeler R., Van Kampen K.R. \& James L.F. (ed.), Effetcs of Poisonous Plants on Livestock. Acedemic Press, New York. 600p.

Fighera R.A. \& Barros C.S.L. 2004. Systemic granulomatous disease in Brazilian cattle grazing pasture containing vetch (Vicia spp). Vet. Human Toxicol. 46:62-66.

Fighera R.A., Rozza D.B., Piazer J.V., Copetti M.V., Irigoyen L.F. \& Barros C.S.L. 2003. Pneumonia intersticial em bovinos associada à ingestão de batatadoce (Ipomoea batatas) mofada. Pesq. Vet. Bras. 23:161-166.

Fighera R.A., Souza T.M. \& Barros C.S.L. 2005. Lesões de pele em bovinos com doença granulomatosa sistêmica associada ao pastoreio de ervilhaca (Vicia spp.). Ciência Rural, Santa Maria, 35:406-411.

Fighera R.A., Souza T.M., Rissi D.R. \& Barros C.S.L. 2006. Intoxicação por samambaia (Pteridium aquilinum) em bovinos: aspectos clínicos, hematológicos e patológicos. In: Trabalhos apresentados no Encontro Nacional de Laboratórios de Diagnóstico Veterinário, Campo Grande, MS, p.29. (Resumo)

Garmatz S.L., Raffi M.B., Rodrigues A., Irigoyen L.F. \& Barros C.S.L. 2001. Intoxicação espontânea por brotos de Xanthium spp em bovinos no Rio Grande do Sul. In: Resumos $10^{\circ}$ Encontro Nacional de Patologia Veterinária, Pirassununga, SP, p.56. (Resumo)

Gava A. 1993. Intoxicação por Pteridium aquilinum, p.247-258. In: Riet-Correa F., Méndez M.C. \& Schild A.L. (ed.), Intoxicações por Plantas e Micotoxicoses em Animais Domésticos. Hemisfério Sul do Brasil, Pelotas. 340p.

Gava A. \& Barros C.S.L. 2001. Field observations of Ateleia glazioviana poisoning in cattle in southern Brazil. Vet. Human Toxicol. 43:37-41.
Gava A., Barros C.S.L., Pilati C., Barros S.S., Mori A.M. 2001. Intoxicação por Ateleia glazioviana (Leg. Papilionoideae) em bovinos. Pesq. Vet. Bras. 21:4959.

Ilha M.R.S., Riet-Correa F. \& Barros C.S.L. 2001. Síndrome distérmica (hipertermia) em bovinos associada à intoxicação por Claviceps purpurea. Pesq. Vet. Bras. 21:81-86.

Ilha M.R.S., Loretti A.P. \& Barros C.S.L. 2003. Hyperthermic syndrome in dairy cattle associated with consumption of ergots of Claviceps purpurea in southern Brazil. Vet. Human Toxicol. 45:140-145.

James L.F. 1978. Overview of poisonous plants problems in the United States, p.3-5. In: Keeler R., Van Kampen K.R. \& James L.F. (ed.), Effetcs of Poisonous Plants on Livestock. Academic Press, New York. 600p.

Karam F.S.C., Soares M.P., Haraguchi M., Riet-Correa F., Méndez M.C. \& Jarenkow J.A. 2004. Aspectos epidemiológicos da seneciose na região sul do Rio Grande do Sul. Pesq. Vet. Bras. 24:191-198.

Langohr I.M., Gava A., Barros C.S.L. 2005. Intoxicação por Baccharidastrum triplinervium (Asteraceae) em bovinos. Pesq. Vet. Bras. 25:235-238.

Lemos R.A., Barros C.S.L., Salles M.S., Barros S.S. \& Peixoto P.V. 1993. Intoxicação espontânea por Amaranthus spinosus (Amaranthaceae) em bovino. Pesq. Vet. Bras. 13:25-34.

Masuda E.K., Rissi D.R., Barros R.R. \& Barros C.S.L. 2005. Epidemiologia, sinais clínicos e patologia da intoxicação por Senecio spp. em bovinos no Rio Grande do Sul. Arq. Bras. Med. Vet. Zootec. 57:65-65.

Ministério da Agricultura Pecuária e Abastecimento (MAPA) 2006. Disponível na internet: http://www.agricultura.gov.br/pls/portal/docs/PAGE/MAPA/ ESTATISTICAS/PECUARIA/3.1.XLS

Pedroso P.M.O., Raymundo D.L., Guagnini F.S., Oliveira E.C., Corrêa A.M.R., Colodel E.M. \& Driemeier D. 2005. Intoxicações por plantas e substâncias químicas em ruminantes diagnosticadas no Setor de Patologia Veterinária da UFRGS no período de 1997-2004. Arq. Bras. Med. Vet. Zootec. 57:74-75.

Peixoto P.V., França T.N., Barros C.S.L. \& Tokarnia C.H. 2003. Aspectos histopatológicos da hematúria enzoótica bovina no Brasil. Pesq. Vet. Bras. 23:65-81.

Rech R.R., Fighera R.A., Oliveira F.N. \& Barros C.S.L. 2004. Meningoencefalite granulomatosa em bovinos em pastoreio de ervilhaca (Vicia spp.). Pesq. Vet. Bras. 24:169-172.

Rech R.R., Rissi D.R., Rodrigues A., Pierezan F., Piazer J.V.M., Kommers G.D. \& Barros C.S.L. 2006. Intoxicação por Solanum fastigiatum (Solanaceae) em bovinos: históricos, sinais clínicos e morfometria das lesões cerebelares. Pesq. Vet. Bras. 26:123-132.

Rech R.R., Rodrigues A., Rissi D.R., Riet-Correa F. \& Barros C.S.L. 2007. Poisonous plants affecting the central nervous system (CNS) of cattle in Brazil, p.101-105. In: Panter, K.E., Wierenga, T.L. \& Pfister, J.A. (ed.), Poisonous Plants: Global research and solutions. CAB International, Wallingford, Oxfordshire, UK.

Riet-Correa F. \& Medeiros R.M.T. 2001. Intoxicações por plantas em ruminantes no Brasil e no Uruguai: importância econômica, controle e riscos para a saúde pública. Pesq. Vet. Bras. 21:38-42.

Riet-Correa F. \& Méndez M.C.1993. Introdução ao estudo das plantas tóxicas, p.1-19. In: Riet-Correa F., Méndez M.C. \& Schild A.L. (ed.), Intoxicações por Plantas e Micotoxicoses em Animais Domésticos. Hemisfério Sul do Brasil, Pelotas. 340p.

Riet-Correa F., Schild A.L., Méndez M.C., Tavares A.S. \& Rodrigues J.O. 1983. Intoxicação por Claviceps paspali em bovinos no Rio Grande do Sul. Pesq. Vet. Bras. 3:59-65.

Riet-Correa F., Méndez M.C. \& Schild A.L. 1993. Intoxicações por Plantas e Micotoxicoses em Animais Domésticos. Hemisfério Sul do Brasil, Pelotas. 340 p.

Riet-Correa F., Méndez M.C., Barros C.S.L. \& Gava A. 1994. Poisonous plants of Rio Grande do Sul, p.13-18. In: Colegate S.M. \& Dorling P.R. (ed.), PlantAssociated Toxins: Agricultural, phytochemical and ecological aspects. $C A B$ International, Wallingford, Oxfordshire, UK.

Riet-Correa F., Barros C.S.L. \& Schild A.L. 2004. Intoxication by Ramaria flavobrunnescens in domestic animals, p. 488-493. In: Acamovic T., Stewart C.S. 
\& Pennycott T.W. (ed.), Poisonous Plants and Related Toxins. CAB International, Wallingford, Oxfordshire, UK.

Rissi D.R., Driemeier D., Silva M.C., Barros R.R. \& Barros C.S.L. 2007. Poisonous plants producing acute hepatic disease in Brazilian cattle, p.7276. In: Panter K.E., Wierenga T.L. \& Pfister J.A. (ed.), Poisonous Plants: Global research and solutions, $\mathrm{CAB}$ International, Wallingford, Oxfordshire, UK.

Rissi D.R., Rech R.R., Fighera R.A., Cagnini D.Q., Kommers G.D. \& Barros C.S.L. 2005. Intoxicação espontânea por Baccharis coridifolia em bovinos. Pesq. Vet. Bras. 25:111-114.

Rozza D.B., Raymundo D.L., Corrêa A.M.R., Seitz A.L., Driemeier D. \& Colodel E.M. 2006. Intoxicação espontânea por Baccharis coridifolia em ovinos. Pesq. Vet. Bras. 26:21-25.

Sanches A.W.D., Langohr I.M., Stigger A.L. \& Barros, C.S.L. 2000. Doenças do sistema nervoso central em bovinos no sul do Brasil. Pesq. Vet. Bras. 20:113-118.

Schild A.L. 2006. Comunicação pessoal (Laboratório Regional de Diagnóstico, Universidade Federal de Pelotas). E-mail: alschild@zaz.com.br

Schuster J.L. 1978. Poisonous plants management problems and control measures on U.S. rangelands, p.23-34. In: Keeler R., Van Kampen K.R. \& James L.F. (ed.), Effetcs of Poisonous Plants on Livestock. Acedemic Press, New York. 600p.
Souto M.A.M., Kommers G.D., Barros C.S.L., Piazer J.V.M., Rech R.R., RietCorrea F. \& Schild A.L. 2006a. Neoplasias do trato alimentar superior de bovinos associadas ao consumo espontâneo de samambaia (Pteridium aquilinum). Pesq. Vet. Bras. 26:112-122.

Souto M.A.M., Kommers G.D., Barros C.S.L., Rech R.R. \& Piazer J.V.M. 2006b. Neoplasmas da bexiga associados à hematúria enzoótica bovina. Ciência Rural, Santa Maria, 36:1647-1650.

Souza M.V. \& Graça D.L. 1993. Intoxicação crônica por Pteridium aquilinum (L.) Kuhn (Polypodiaceae) em bovinos. Ciência Rural, Santa Maria, 23:203207.

Tokarnia C.H., Döbereiner J., Silva M.F. 1979. Plantas Tóxicas da Amazônia a Bovinos e Outros Herbívoros. Instituto Nacional de Pesquisas da Amazônia, Manaus. 95p.

Tokarnia C.H., Döbereiner J. \& Peixoto P.V. 2000. Plantas Tóxicas do Brasil. Editora Helianthus, Rio de Janeiro. 320p.

Torres M.B., Kommers G.D., Dantas A.F. \& Barros C.S.L. 1997. Redroot pigweed (Amaranthus retroflexus) poisoning in cattle in southern Brazil. Vet. Human Toxicol. 3:94-96.

Whittem J.H.1978. Poisonous plants problems in Australia, p.7-10. In: Keeler R., Van Kampen K.R. \& James L.F. (ed.), Effetcs of Poisonous Plants on Livestock. Academic Press, New York. 600p. 